\title{
Secondary Electron Production at the SNS Storage Ring Collimator*
}

\author{
S.Y. Zhang \\ AGS Department, Brookhaven National Laboratory, Upton, NY, USA
}

\begin{abstract}
Secondary electron (SE) production is briefly reviewed. If the collimator of the SNS storage ring allows proton beam scraping to take place, the electron yield might be quite large.
\end{abstract}

At the AGS Booster, by steering the $A u^{31+}$ ion beam into the electrostatic inflector, beam scraping effect on SE production is studied.

The results of this experiment can be translated into the situation of proton beam scraping at the SNS collimator. It seems sufficient to support a new look of the SNS ring collimator design.

\section{Secondary Electron Production}

In secondary electron emission, the electronic stopping (Coulomb collision) is dominant if the projectile velocity is larger than the Bohr velocity $2.18 \times 10^{8} \mathrm{~cm} / \mathrm{s}(\beta=0.0073)$. If the primary ion, proton, or electron have the same velocity, the kinetics of the collision is very similar [1,2]. The Seiler model shows that the peak SE production energy of projectile is around $E_{k} \approx 0.9 \mathrm{MeV} / u(\beta=0.044)$. Also according to this model, the SE production rate at the SNS beam energy, $E_{k} \approx 1.0 \mathrm{GeV} / u(\beta=0.875)$, is about $10 \%$ of the peak yield.

Since the electronic stopping power of the target is approximately proportional to $q^{2}$, where $q$ is the charge state of the projectile, it was believed that the SE yield $Y$ also has a $q^{2}$ dependence [5,6]. However, experimental results have shown that it is more likely a $q^{1.7}$ dependence [7].

Probably the most profound factor in SE emission is the projectile scraping effect. Only the excited electrons near the surface have a chance to escape, and a major part of stopping power of a grazing projectile is deposited on the surface. In [3], this dependence is estimated as a factor $(\cos \theta)^{-n}$, where a

\footnotetext{
* Work performed under the auspices of the US Dept. of Energy
}

perpendicular incident has an angle $\theta=0$, and a range of the index $0.8 \leq n \leq 15$ is indicated. The complication of this mechanism, both theoretically and experimentally, in fact prohibits any accurate account on this factor.

Following an experimental observation, in [8], it was calculated that $Y \approx 200$ for a grazing proton at the PSR of LANL, $\beta=0.841$. The electron collection there seems in agreement with this yield.

If the collimator of the SNS storage ring allows proton beam scraping to take place, the electron yield will probably be around 200 . Note that this yield is about 1,000 times higher than the yieid that has been theoretically and experimentally confirmed, without the functioning of the scraping effect.

To be more confident with the necessity to eliminate proton beam scraping on the surface of the collimator, an experiment was performed at the AGS Booster to study the beam scraping effect on the SE production.

\section{Experiment at the AGS Booster}

By horizontally steering the $A u^{31+}$ ion beam into the electrostatic inflector, which guides the ion beam from the Tandem transfer line into the Booster orbit, a situation of beam scraping on the inflector surface at different angles is created. Since the projectile energy and charge state effects on the SE production are known, this scraping study could be a useful reference for the SNS collimator electron production.

The inflector has a horizontal aperture $17 \mathrm{~mm}$, and is normally charged at $24 K V$. The capacitance at the inflector is about $300 p f$, and the charging resistance is $1 M \Omega$. The anode of the inflector is grounded, therefore, the cathode carries a voltage of $-24 K V$. By steering the ion beam into the cathode, the electrons there may escape from the surface, then these electrons are expelled by the cathode. By observing the cathode voltage, therefore, the secondary electron emission can be estimated.

The gold ions from the Tandem to Booster transfer line (TtB) carry a positive charge of 31 . The kinetic energy is $E_{k} \approx 0.9 \mathrm{MeV} / u(\beta=0.044)$, which happens to be the peak production energy of SE. 
In a normal running condition, the Tandem to Booster transfer line horizontal dipole 29T $\mathrm{DH} 2$ upstream the inflector was set at $-0.55 \mathrm{~A}$. The beam full width half magnitude (FWHM) size was $4 \mathrm{~mm}$. It is believed that during $670 \mu \mathrm{s}$ multiturn injection period, beam scraping at either the anode or the cathode causes a voltage drop at the inflector. This voltage decline is almost invisible at low intensity, and it is about $300 \mathrm{~V}$ at the high intensity injection. After the stacking, the inflector voltage is recovered by the charging current of the power supply. The high intensity of gold beam injection usually implies more than $3 \times 10^{9}$ ions per pulse.

By setting the $\mathrm{DH} 2$ current at $-3.76 \mathrm{~A}$, $-3.96 A$, and $-4.16 A$, the detected inflector voltage variation is shown in Fig.l. For convenience, the voltage has been offset by $-24 K V$. We observe that at the end of injection period, the inflector cathode voltage is raised by $2 K V, 8.6 K V$, and $7 K V$, respectively. In other words, the cathode voltage becomes $-22 \mathrm{KV}$, $-15.4 K V$, and $-17 K V$ at the end of stacking, respectively.
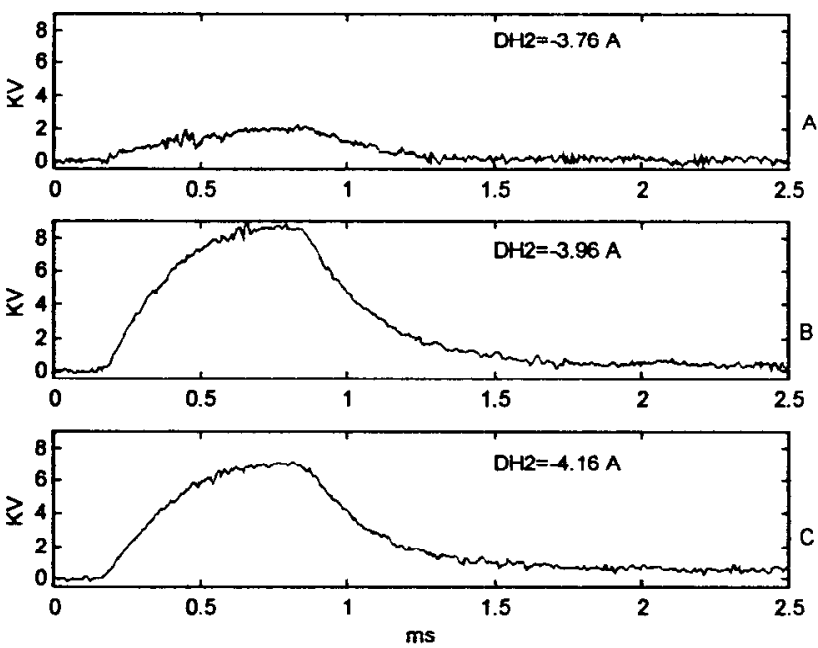

Fig. I. Beam Scraping Induced Voltage on the Inflector

A simple model is used to explain the results. A constant voltage source, at $V_{1}=-24 K V$, charges the inflector through $R=1 M \Omega$. The inflector itself is represented by a capacitance of $C=300 p f$, and its voltage is $V_{2}$. The ion beam generated $\mathrm{SE}$ production is modeled as a current source $I$. At the beginning of stacking, we have $V_{1}=V_{2}=-24 K V$ and $I=0$. Once the ion beam is steered into the cathode, $I \neq 0$. The inflector cathode voltage rises, which in turn induces the charging current through $R$. At the end of the stacking, once again $I=0$. The inflector cathode voltage is recovered by the charging current. The following equation can be used to describe this model.

$$
V_{2}=V_{2,0}+\frac{1}{C} \int\left(\frac{V_{1}-V_{2}}{R}-I\right) d t
$$

where $V_{2,0}=-24 K V$ is the static cathode voltage. Using the detected $V_{2}$, we find $I$, which is then used to get the $\mathrm{SE}$ yield. This is,

$$
I(t)=\frac{V_{1}-V_{2}}{R}-C \frac{d V_{2}}{d t}
$$

By fitting to $V_{2}$, we find that both the rising and falling of this voltage are exponential. The time constant of the rising is $\tau_{\text {rise }}=2 \times 10^{-4}$ sec. , and the falling time constant is $\tau_{\text {fall }}=3 \times 10^{-4} \mathrm{sec}$. This is shown in Fig.2, where the amplitude of $V_{2}$ is normalized to unity. The falling time constant confirms that $R=1 M \Omega$ and $C=300 \mathrm{pf}$,

$$
\tau_{\text {fall }}=R C=3 \times 10^{-4} \mathrm{sec} .
$$

On the other hand, the fit of the rising voltage, during the stacking period, requires $I$ to be time dependent.

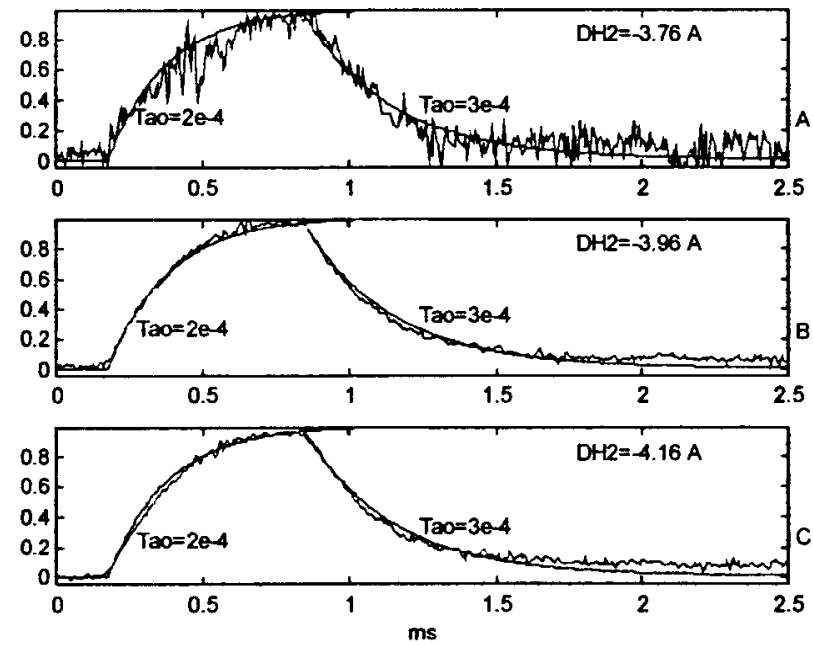

Fig.2. Time Constants of the Rising and Falling Voltage on the Inflector Cathode

Take the case that the $\mathrm{DH} 2$ current of $-3.96 \mathrm{~A}$ as an example. The current $I$ is calculated using (2) and the measured $V_{2}$. The result is shown in Fig.3. At the beginning of stacking, this current is $13.3 \mathrm{~mA}$ and at the end of stacking, it is reduced to $9 m A$. This variation is not a surprise. However, a convincing explanation of this is difficult to reach. Among possible reasons, the most noticeable ones are: 
1. Electrostatic potential that deflects the projectile. In our case, the inflector voltage has been dropped significantly during stacking. In fact, shortly after the beginning of stacking, most ions in the beam have been completely deflected and hit the anode.

2. Electron-depletion effect. At $133 \mathrm{~mA}$, the electrons escape the surface at a rate of $83 \times 10^{16}$ per second. Depending on the thickness of the electron exciting layer, depletion might take place.

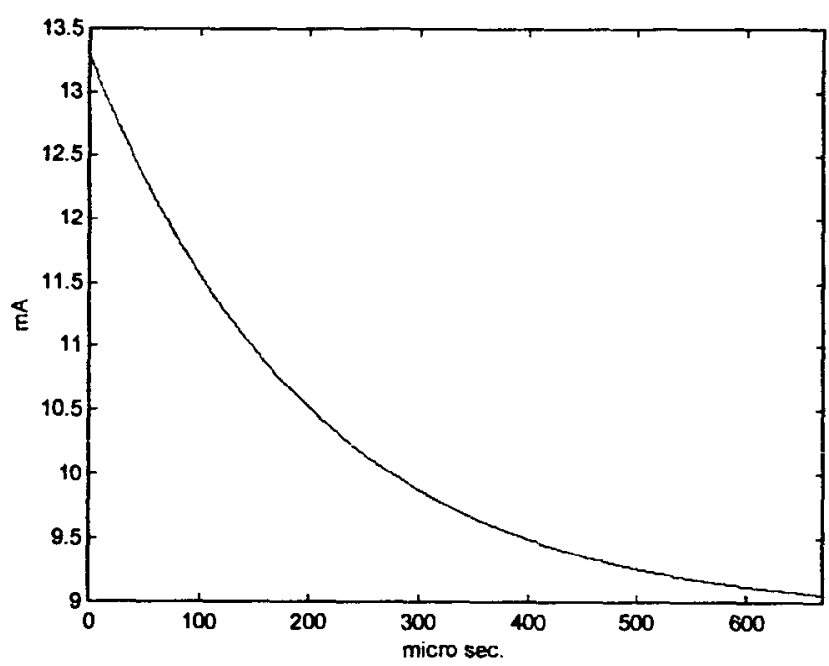

Fig.3. Equivalent Current due to the Beam Scraping

To estimate the $\mathrm{SE}$ yield due to beam scraping, therefore, the peak current of $13.3 \mathrm{~mA}$ can be used.

A nontrivial question is how much scraping ions are responsible for these secondary electrons. Let us assume that the $\mathrm{DH} 2$ current $-0.55 \mathrm{~A}$ places the beam in the center of the inflector aperture, and the current $-4 A$ steers the beam center into the cathode. Therefore, the current of $3.45 \mathrm{~A}$ steers the beam horizontally by half the inflector aperture, $8.5 \mathrm{~mm}$. It is shown in Fig. 1 that at the $\mathrm{DH} 2$ current of $-3.76 \mathrm{~A}$, the cathode voltage is raised by $2 K V$ at the end of stacking. Decreasing the current by $02 \mathrm{~A}$, the cathode voltage is raised by $8.6 K V$, and another $0.2 A$, it is $7 K V$. DH2 current of $02 \mathrm{~A}$ implies a $0.5 \mathrm{~mm}$ beam horizontal position shift. In comparison, we note that the beam FWHM size was $4 \mathrm{~mm}$, and $\sigma=1.7 \mathrm{~mm}$.

\section{Secondary Electron Yield}

Let us take $20 \%$ of the beam per pulse to be responsible for the scraping effect in producing SE. This is $6 \times 10^{8}$ ions. Thus, we have the SE yield per lost gold ion,

$$
Y_{1}=93 \times 10^{4}
$$

To translate this yield intc ine SNS situation, we take the SE production rate at $1 \mathrm{GeV}$ as $10 \%$ of that in the experiment. Also we assume the projectile charge state dependence as $q^{1.7}=31^{1.7}=343$. Then, the SE yield shown in (4) is,

$$
Y_{2}=\frac{Y_{1}}{10 \times 343}=27
$$

This yield is smaller than the one estimated in [8], however, it is much larger than the one observed in experiments without scrapping effect. For instance, see [9]. Note also that the early estimate of the SNS collimator SE yield was 0.25 to 2 , depending on the collimator edge angle [10].

\section{Conclusion}

The experiment performed at the AGS Booster, using $A u^{31+}$ ion beam to scrape on the electrostatic inflector, has shown the significance of the scraping effect on the secondary electron production. The result of this experiment seems sufficient to support a new look at the SNS ring collimator design [11].

Recent study performed at the BNL Tandem has confirmed the glancing effect of SE production. Using the serrated plate with a sawtooth surface, the SE production reduced dramatically [12].

\section{Acknowledgment}

The author would like to thank L.A. Ahrens, C.J. Gardner, and A.V. Soukas for valuable discussions and help in the experiment.

\section{References}

1. A.B. Lapnnsky, J. Appl. Phys. 34, 1568 (1963).

2. J.E. Borovsky, D.J. McComas and B.L. Barraclough, Nucl. Instrum. Methods, B30, 191 (1988).

3. H. Seiler, J. Appl. Phys. 54, R1 (1983).

4. S.Y. Zhang, AGS Tech. Note, No. 477, BNL, May, (1998).

5. H.A. Bethe and J. Ashkin, Experimental Nuclear Physics, Vol. I, Edited by E. Segre (Wiley, New York, 1953).

6. J. Lindhard, Nucl. Instrum. Methods, 132, 1 (1976).

7. J.E. Borovsky and D.M. Suszcynsky, Phys. Rev. A43, 1416 (1991).

8. M. Plum, PSR-95-001, LANL, Feb. (1995).

9. O. Grobner, LHC Project Report, 127 (1997).

10. L.N. Blumberg, Jan. 1998, unpublished.

11. H. Ludewig, private communication.

12. P. Thieberger et. al, private communication. 